Proceeding Series of the Brazilian Society of Computational and Applied Mathematics

\title{
Explorando Analiticamente o Modelo de Rede Neural de Wilson-Cowan
}

\author{
Lucas Leocádio das Neves ${ }^{1}$ \\ Programa de Pós-graduação em Engenharia Elétrica e Computação da Universidade \\ Presbiteriana Mackenzie, São Paulo, SP

\section{Luiz Henrique Alves Monteiro²} \\ Escola de Engenharia da Universidade Presbiteriana Mackenzie e Escola Politécnica da \\ Universidade de São Paulo, São Paulo, SP
}

\begin{abstract}
Resumo. Considere duas populações de neurônios, uma excitatória e outra inibitória, de modo que essas populações estão intra e interconectadas. O modelo de Wilson-Cowan descreve a evolução temporal da atividade dessa rede neural. Aqui, estuda-se analiticamente uma versão desse modelo e mostra-se que tal atividade pode ser oscilatória.
\end{abstract}

Palavras-chave. Ciclo-limite, estabilidade, modelo de Wilson-Cowan, rede neural, sistemas dinâmicos.

\section{Introdução}

Em diversos estudos em neurociência, assume-se que o elemento básico não é um único neurônio, mas é, sim, um conjunto de neurônios. Como exemplo, pode-se citar a coluna cortical, tipicamente formada por dezenas de neurônios, e que pode ser considerada como a unidade de processamento do córtex cerebral [1]. Um modelo famoso que descreve a evolução temporal da atividade (elétrica) de populações neuronais é aquele que foi proposto por Hugh R. Wilson e Jack D. Cowan [11], em 1972. Tal modelo consiste de duas equações diferenciais de primeira ordem não lineares, que representam as interações entre duas populações de neurônios que são distinguidas pelo fato de que seus axônios terminam em sinapses que são ou excitatórias ou inibitórias. Assim, as variáveis do modelo de Wilson-Cowan são as atividades das populações de neurônios excitatórios e de neurônios inibitórios, que variam com o passar do tempo. Essas populações podem receber estímulos advindos de outras estruturas neurais, musculares, sensoriais.

$171457704 @$ mackenzista.com.br

2 luizm@mackenzie.br, luizm@usp.br 
Conjectura-se que o sincronismo entre disparos neuronais seja fundamental para a realização bem sucedida de funções cognitivas que necessitam da interação coordenada de neurônios distribuídos em diversas áreas do cérebro. Como exemplo de tais funções, podem-se citar [6,9]: a correta percepção de estímulos visuais e auditivos, a formação de memória, a capacidade de se concentrar durante a resolução problemas, a realização de "insights" (a determinação de um resultado de modo repentino e intuitivo). Portanto, neurônios sadios se sincronizam.

Como atividade oscilatória é usualmente observada no sistema nervoso de animais $[1,4]$ e como tal tipo de atividade pode estar relacionada à emergência de sincronismo [6,9], neste trabalho investiga-se analiticamente a existência de solução oscilatória autossustentada numa versão do modelo de Wilson-Cowan.

Este trabalho sobre neurodinâmica está assim organizado. Na Seção 2, descreve-se a versão do modelo de Wilson-Cowan aqui investigada. Na Seção 3, obtêm-se resultados analíticos sobre atividades estacionária e oscilatória. Na Seção 4, apresentam-se simulações numéricas, com a intenção de ilustrar os resultados da Seção 3. Na Seção 5, discutem-se a contribuição e a limitação deste trabalho.

\section{O modelo de Wilson-Cowan}

O modelo de Wilson-Cowan tem sido empregado em vários estudos teóricos sobre dinâmica de redes neurais, com o objetivo de tentar entender os padrões de disparos observados experimentalmente em sistemas nervosos reais [2].

Considere que $\mathrm{x}(\mathrm{t})$ e $\mathrm{y}(\mathrm{t})$ representem a atividade da população de neurônios excitatórios e a atividade da população de neurônios inibitórios, respectivamente. Suponha que $x(t)=0$ e $y(t)=0$ correspondam à atividade basal, de modo que valores negativos de $x(t)$ e $y(t)$ indicam uma atividade abaixo desse nível basal. Segundo Wilson e Cowan, a evolução temporal de $x(t)$ e $y(t)$ pode ser descrita pelas seguintes equações diferenciais $[7,11]$ :

$$
\begin{aligned}
& \mathrm{dx}(\mathrm{t}) / \mathrm{dt}=\mathrm{f}_{1}(\mathrm{x}(\mathrm{t}), \mathrm{y}(\mathrm{t}))=-\mathrm{ax}(\mathrm{t})+\mathrm{S}(\mathrm{wx}(\mathrm{t})-\mathrm{by}(\mathrm{t})+\mathrm{M}(\mathrm{t})) \\
& \mathrm{dy}(\mathrm{t}) / \mathrm{dt}=\mathrm{f}_{2}(\mathrm{x}(\mathrm{t}), \mathrm{y}(\mathrm{t}))=-\mathrm{dy}(\mathrm{t})+\mathrm{S}(\mathrm{cx}(\mathrm{t})-\mathrm{ey}(\mathrm{t})+\mathrm{N}(\mathrm{t}))
\end{aligned}
$$

em que $\underline{a}$ e $\underline{\mathrm{d}}$ são as constantes de taxa de decaimento (exponencial) natural da atividade da população excitatória e da população inibitória, respectivamente; $\underline{w}$ é a intensidade da autoconexão da população excitatória, e é a intensidade da autoconexão da população inibitória, $\underline{b}$ é a intensidade do acoplamento partindo da população inibitória e chegando à população excitatória, e $\underline{c}$ é a intensidade do acoplamento partindo da população excitatória e chegando à população inibitória. Esses seis parâmetros $\mathrm{a}, \mathrm{b}, \mathrm{c}, \mathrm{d}, \mathrm{e}, \mathrm{w}$ são números positivos. As entradas $\mathrm{M}(\mathrm{t})$ e $\mathrm{N}(\mathrm{t})$ representam estímulos oriundos, por exemplo, de outras estruturas neurais, e que afetam, respectivamente, as populações excitatória e inibitória em estudo.

De acordo com os autores do modelo [11], a função S(z) é "qualquer" função sigmoidal; ou seja, qualquer função com um único ponto de inflexão, monotonamente crescente e que satura para $\mathrm{z} \rightarrow-\infty$ e para $\mathrm{z} \rightarrow+\infty$. A função sigmoidal por eles escolhida foi $\mathrm{S}(\mathrm{z})=1 /(1+\exp (-\mathrm{z}))$; entretanto, essa escolha de $\mathrm{S}(\mathrm{z})$ dificulta investigações analíticas. 
Uma função sigmoidal que possibilita estudos analíticos é $\mathrm{S}(\mathrm{z})=\mathrm{z} / \sqrt{1+\mathrm{z}^{2}}$. Essa função foi usada por Monteiro e colegas [7] na análise das Equações (1)-(2) tomando e=0. Aqui, supõe-se que e $\neq 0, a \ll 1$ e $d \ll 1$. Os resultados apresentados a seguir são originais.

\section{Resultados analíticos}

Considere $M(t)=M$ e $N(t)=N$, com $M$ e $N$ constantes. Tais entradas constantes podem representar valores médios dos estímulos recebidos pelas populações neurais em questão. Nesse caso, o sistema apresenta soluções estacionárias, ou seja, soluções $(\mathrm{x}(\mathrm{t}), \mathrm{y}(\mathrm{t}))=\left(\mathrm{x}^{*}, \mathrm{y}^{*}\right)$ com $x^{*}$ e $y^{*}$ constantes. Tais constantes são determinadas a partir de $f_{1}\left(x^{*}, y^{*}\right)=0$ e $f_{2}\left(x^{*}, y^{*}\right)=0$. No espaço de estados $\mathrm{x} \times \mathrm{y}$, soluções estacionárias correspondem a pontos de equilíbrio $[3,8]$. Se a«1 e d«1, verifica-se que há um único ponto de equilíbrio, cujas coordenadas são:

$$
\begin{aligned}
& \mathrm{x}^{*}=[(\mathrm{e}+\mathrm{d}) \mathrm{M}-\mathrm{bN}] /[(1-\delta) \mathrm{bc}] \\
& \mathrm{y}^{*}=[\mathrm{cM}-(\mathrm{w}-\mathrm{a}) \mathrm{N}] /[(1-\delta) \mathrm{bc}]
\end{aligned}
$$

$\operatorname{com} \delta=(\mathrm{w}-\mathrm{a})(\mathrm{d}+\mathrm{e}) /(\mathrm{bc})$.

A estabilidade local de um ponto de equilíbrio $\left(\mathrm{x}^{*}, \mathrm{y}^{*}\right)$ pode ser inferida a partir dos autovalores da matriz jacobiana J, que é obtida linearizando o sistema em torno desse ponto $[3,8]$; ou seja:

$$
\mathrm{J}=\left(\begin{array}{ll}
\partial \mathrm{f}_{1}(\mathrm{x}, \mathrm{y}) / \partial \mathrm{x} & \partial \mathrm{f}_{1}(\mathrm{x}, \mathrm{y}) / \partial \mathrm{y} \\
\partial \mathrm{f}_{2}(\mathrm{x}, \mathrm{y}) / \partial \mathrm{x} & \partial \mathrm{f}_{2}(\mathrm{x}, \mathrm{y}) / \partial \mathrm{y}
\end{array}\right)(\mathrm{x}, \mathrm{y})=\left(\mathrm{x}^{*}, \mathrm{y}^{*}\right)
$$

De acordo com o teorema de Hartman-Grobman [3,8], $\left(\mathrm{x}^{*}, \mathrm{y}^{*}\right)$ é ponto de equilíbrio localmente assintoticamente estável se os autovalores $\lambda$ de J possuem parte real negativa. Esses autovalores são determinados a partir $\operatorname{de} \operatorname{det}(\mathbf{J}-\lambda \mathbf{I})=0$, sendo I a matriz identidade $[3,8]$. Para um sistema de segunda ordem, os autovalores são as raízes de $\lambda^{2}-\mathrm{T} \lambda+\Delta=0$, em que $\mathrm{T}$ é o traço e $\Delta$ é o determinante da matriz J. Esse polinômio tem raízes com parte real negativa (ou seja, o ponto de equilíbrio correspondente é assintoticamente estável) somente se $\mathrm{T}<0$ e $\Delta>0[3,8]$. Para a« 1 e d $\ll 1$, mostra-se que:

$$
\begin{aligned}
& \mathrm{T}=\mathrm{w}-(\mathrm{a}+\mathrm{d}+\mathrm{e}) \\
& \Delta=(1-\delta) \mathrm{bc}
\end{aligned}
$$

Portanto, $\left(\mathrm{x}^{*}, \mathrm{y}^{*}\right)$ é localmente assintoticamente estável se $\mathrm{w}<(\mathrm{a}+\mathrm{d}+\mathrm{e})$ e $0<\delta<1$. Como T e $\Delta$ independem de $\mathrm{M}$ e $\mathrm{N}$, então entradas constantes não alteram a estabilidade do ponto de equilíbrio dado pelas Equações (3) e (4). Assim, os parâmetros que determinam a estabilidade da solução estacionária $(x(t), y(t))=\left(x^{*}, y^{*}\right)$ são apenas aqueles relacionados com $\mathrm{o}$ acoplamento das populações neuronais.

Ciclo-limite (ou seja, oscilação autossustentada) pode passar a ser solução de um sistema dinâmico de tempo contínuo via bifurcação de Hopf [3,8]. O sistema de Equações (1) e (2) sofre uma bifurcação de Hopf para a combinação de valores de parâmetros tais que $\mathrm{T}=0 \mathrm{e}$ $\Delta>0$. Portanto, o valor crítico $\mathrm{w}_{\mathrm{c}}$ da intensidade da autoconexão da população excitatória, 
associada ao surgimento de ciclo-limite, é $\mathrm{w}_{\mathrm{c}}=(\mathrm{a}+\mathrm{d}+\mathrm{e})$. Considerando a população inibitória, o valor crítico $e_{c}$ da respectiva autoconexão é $e_{c}=w-(d+a)$. Assim, há ciclo-limite se $\mathrm{w}>\mathrm{w}_{\mathrm{c}}$ ou se, para $\mathrm{w}>0$, então $\mathrm{e}<\mathrm{e}_{\mathrm{c}}$. Os valores das constantes $\underline{\mathrm{b}}$ e $\underline{\mathrm{c}}$, que representam as conexões entre as duas populações, não afetam o traço $\mathrm{T}$; logo, o sistema não pode sofrer bifurcação de Hopf com a variação das intensidades dessas conexões.

Note que, com entradas constantes e na ausência da autoconexão excitatória $(w=0)$, as atividades das populações neuronais não podem ser oscilatórias. Entretanto, na presença dessa autoconexão, tais atividades podem oscilar espontaneamente, mesmo no caso em que as populações não recebem estímulos periódicos. Esse resultado é relevante, pois redes neurais biológicas podem exibir atividade oscilatória mesmo quando não são estimuladas [4].

\section{Simulações numéricas}

Com a intenção de ilustrar a análise apresentada na seção anterior, nesta seção são mostradas figuras geradas por meio da resolução numérica as Equações (1) e (2), partindo da condição inicial $(\mathrm{x}(0), \mathrm{y}(0))=(0,0)$. Para isso, utilizou-se o método de integração de Runge-Kutta [8] de quarta ordem, com passo de integração igual a 0,01.

As figuras mostram as evoluções temporais de $\mathrm{x}(\mathrm{t})$ e $\mathrm{y}(\mathrm{t})$ e as respectivas trajetórias no espaço de estados $x \times y$. Na Figura $1, a=1 / 10, b=1, c=1, d=1 / 10, e=1 / 2, w=3 / 10$, $M=1$ e $N=0$. Na Figura 2, w=4/5; na Figura 3, e=5/100 (nas Figuras 2 e 3, os demais parâmetros têm os mesmos valores que foram usados na Figura 1).

Na Figura 1, o atrator das Equações (1) e (2) é a solução estacionária $\left(x^{*}, y^{*}\right)=(0,68 ; 1,1)$, que coincide com aquela calculada usando as Equações (3) e (4) (deduzidas supondo a«1 e $\mathrm{d} \ll 1$ ). Nesse caso, das Equações (6) e (7), obtêm-se $\mathrm{T}=-0,40<0$ e $\Delta=0,88>0$, o que corrobora a estabilidade assintótica da solução estacionária.

Nas Figuras 2 e 3, o atrator é um ciclo-limite: na Figura 2 tem-se $w=0,80>w_{c}=0,75$ e, na Figura 3, tem-se e $=0,05<e_{c}=0,1$. Observe que, de fato, o sistema sofre bifurcação de Hopf se a autoconexão excitatória supera $\mathrm{w}_{\mathrm{c}}$, ou se, para $\mathrm{w}>0$, a autoconexão inibitória fica abaixo de $\mathrm{e}_{c}$.
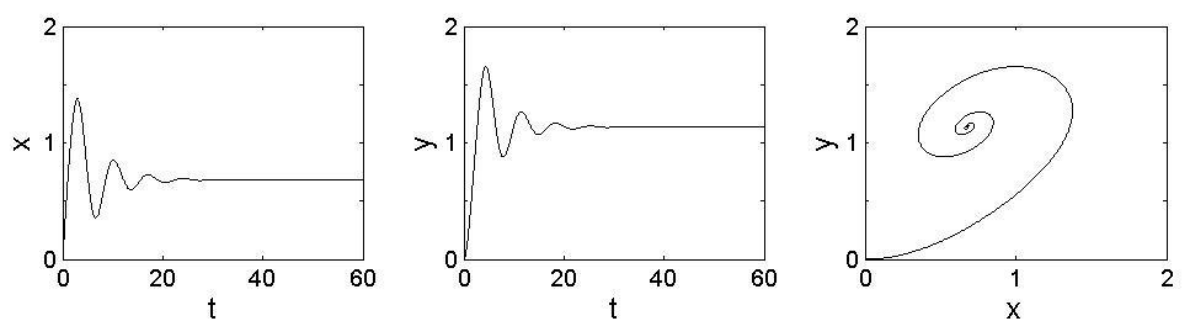

Figura 1: Evoluções temporais de $\mathrm{x}(\mathrm{t})$ e $\mathrm{y}(\mathrm{t})$ e a trajetória correspondente no espaço de estados $\mathrm{x} \times \mathrm{y}$, a partir da condição inicial $(\mathrm{x}(0), \mathrm{y}(0))=(0,0)$. Os valores dos parâmetros são $a=1 / 10, b=1, c=1, d=1 / 10, e=1 / 2, w=3 / 10, M=1$ e $N=0$. 

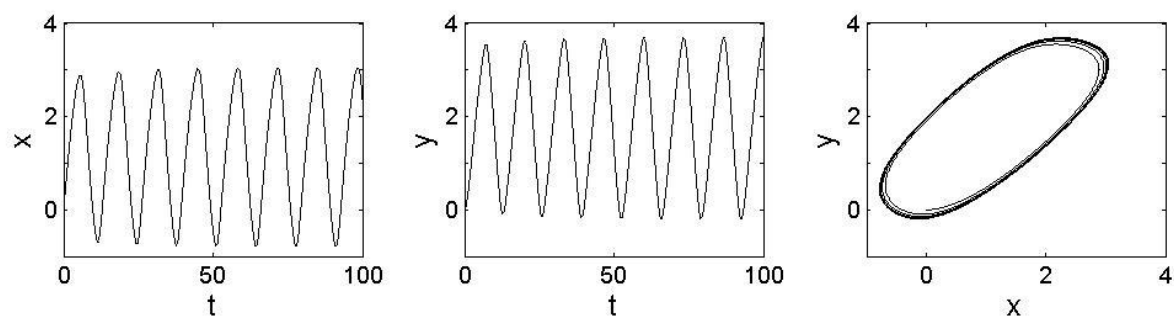

Figura 2: Evoluções temporais de $\mathrm{x}(\mathrm{t})$ e $\mathrm{y}(\mathrm{t})$ e a trajetória no espaço de estados $\mathrm{x} \times \mathrm{y}$ para $\mathrm{w}=4 / 5$ (os demais valores de parâmetros são iguais aos usados na Figura 1).
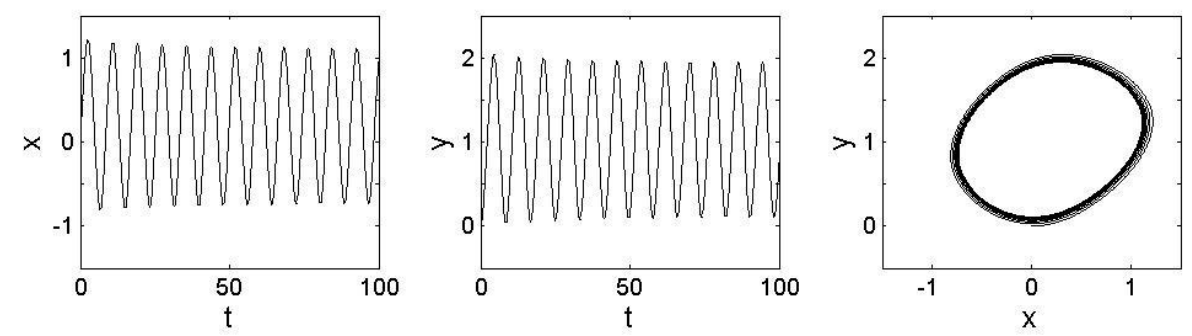

Figura 3: Evoluções temporais de $\mathrm{x}(\mathrm{t})$ e $\mathrm{y}(\mathrm{t})$ e a trajetória no espaço de estados $\mathrm{x} \times \mathrm{y}$ para $\mathrm{e}=5 / 100$ (os demais valores de parâmetros são iguais aos usados na Figura 1).

\section{Conclusões}

Aqui, analisou-se o modelo de Wilson-Cowan, considerando que são "pequenos" os valores das constantes de taxa a e $\underline{\mathrm{d}}$ que expressam o decaimento natural das atividades das populações excitatória e inibitória (isto é, supondo a«1 e d «1). Esse estudo analítico foi possível graças à função sigmoidal convenientemente escolhida (a saber, $\mathrm{S}(\mathrm{z})=$ $\mathrm{z} / \sqrt{1+\mathrm{z}^{2}}$ ). Com essa escolha, mostrou-se que, para entradas constantes, as atividades das populações que formam a rede neural em questão podem convergir para solução estacionária ou para solução periódica. Solução periódica existe somente se a intensidade da autoconexão da população excitatória se encontra acima de um valor crítico (ou seja, se $\mathrm{w}>\mathrm{w}_{\mathrm{c}}=\mathrm{a}+\mathrm{d}+\mathrm{e}$ ). Para $\mathrm{w}>0$, então solução periódica pode ser gerada diminuindo a intensidade da autoconexão da população inibitória a um valor abaixo de um número crítico (ou seja, se $\mathrm{e}<\mathrm{e}_{\mathrm{c}}=\mathrm{w}-\mathrm{d}-\mathrm{a}$ ). Esses resultados sugerem que, para compreender os padrões de atividade de redes neurais biológicas, é relevante determinar experimentalmente em que medida neurônios excitatórios estimulam neurônios excitatórios, e em que medida neurônios inibitórios estimulam neurônios inibitórios.

Numericamente, a dinâmica de redes de Wilson-Cowan acopladas já foi investigada $[5,10]$. Num próximo trabalho, pretende-se acoplar duas redes de Wilson-Cowan (ou seja, dois pares de populações excitatórias e dois pares de populações inibitórias) e investigar, analiticamente, se para parâmetros e entradas constantes, a dinâmica pode se tornar caótica. De fato, caos não pode surgir na rede neural aqui analisada, pois ela é 
descrita por um sistema não linear de segunda ordem que é autônomo, de tempo contínuo, e com campo vetorial contínuo [3,8]. Entretanto, caos pode surgir em sistemas não lineares de tempo contínuo de ordem maior ou igual a três. Registros eletroencefalográficos, tipicamente aperiódicos, são mais similares a um padrão caótico de atividade neural do que a um padrão estacionário ou periódico, que são os que podem existir na versão do modelo aqui analisada.

\section{Agradecimentos}

Lucas L. das Neves agradece à CAPES pela bolsa de mestrado. Luiz H. A. Monteiro agradece ao $\mathrm{CNPq}$ pela bolsa de produtividade.

\section{Referências}

[1] M. F. Bear, B. W. Connors and M. A. Paradiso, Neurociências, Artmed (2002).

[2] A. Destexhe and T. J. Sejnowski, The Wilson-Cowan model, 36 years later, Biol. Cybern., vol. 101, 1-2 (2009).

[3] J. Guckenheimer and P. Holmes, Nonlinear Oscillations, Dynamical Systems, and Bifurcations of Vector Fields, Springer (2002).

[4] P. E. Latham, B. J. Richmond, P. G. Nelson and S. Nirenberg, Intrinsic dynamics in neuronal networks. I. Theory, J. Neurophysiol., vol. 83, 808-827 (2000).

[5] Y. Maruyama, Y. Kakimoto and O. Araki, Analysis of chaotic oscillations induced in two coupled Wilson-Cowan models, Biol. Cybern., vol. 108, 355-363 (2014).

[6] L. H. A. Monteiro, Sistemas Dinâmicos Complexos, Livraria da Física (2014).

[7] L. H. A. Monteiro, M. A. Bussab and J. G. Chauí-Berlinck, Analytical results on a Wilson-Cowan neuronal network modified model, J. Theor. Biol., vol. 219, 83-91 (2002).

[8] L. H. A. Monteiro, Sistemas Dinâmicos, Livraria da Física (2011).

[9] S. H. Strogatz, Sync, Hyperion (2003).

[10] T. Ueta and G. Chen, On synchronization and control of coupled Wilson-Cowan neural oscillators, Int. J. Bifurcat. Chaos, vol. 13, 163-175 (2003).

[11] H. R. Wilson and J. D. Cowan, Excitatory and inhibitory interactions in localized populations of model neurons, Biophys. J., vol. 12, 1-24 (1972). 